\title{
Pathomorphological Assessment Method of Myocardial infaretion Age
}

DOI: 10.17691/stm2017.9.2.15

Received/January 2, 2016

1.A. Shurygina, MD, DSc, Deputy Director on Scientific and Innovation Work'; Chief Researcher';

O. V. Kanya, MD, PhD, Head of the General Pathology Department3;

N.N. Dremina, PhD, Senior Researcher, Laboratory of Pathophysiology of Tissues and Functional Morphology';

M.G. Shurygin, MD, DSc, Head of the Scientific Laboratory Department'; Chief Researcher ${ }^{2}$

Irkutsk Scientific Center of Surgery and Traumatology, 1 Bortsov Revolutsii St., Irkutsk, 664003,

Russian Federation:

2Irkutsk Scientific Center of the Siberian Branch of the Russian Academy of Sciences, 134 Lermontov St.,

Irkutsk, 664033, Russian Federation;

3irkutsk Regional Bureau of Morbid Anatomy, 100 Yubileyny microrayon, Irkutsk, 664079, Russian Federation

The aim of the investigation was to develop a simple and available for practical application method of pathomorphological assessment of myocardial infarction age with a wide range of registered terms.

Materials and Methods. Specimens of the experimental myocardial infarction zone (a model of diathermocoagulation of the rat periconical interventricular artery; $\mathrm{n}=50$ ) in the period from $2 \mathrm{~h}$ to 30 days, and post-mortem specimens of 30 men died of transmural myocardial infarction and post-infarction cardiosclerosis in the period from $6 \mathrm{~h}$ to 30 days were immunohistochemically investigated using antibodies to the matrix metalloproteinase 9 (MMP9).

Results. It has been established that localization of MMP9 in the infarction zone enables determining with a sufficiently high precision the time that passed from infarction onset. In fatal outcomes developed during several hours after infarction, the neutrophil cytoplasm in the infarction zone had intensive color. On day 1-2 a bright color of the extracellular matrix was noted. In later terms (days 3-21) staining of the fibroblastic cell line in the border zone (with maximum on days 7-14) was observed. The dynamics of MMP9 content and its localization in the myocardium of the dead patients corresponds to the data obtained in the experimental investigations.

Conclusion. The proposed method allows pathologists to clearly differentiate myocardial infarction age using pathomorphological investigations.

Key words: myocardial infarction; matrix metalloproteinase 9; MMP9; pathomorphological diagnosis.

A precise determination of the myocardial infarction age is of great significance [1]. Routine methods of anatomico-pathological diagnosis are not always objective and valid, and often do not allow physicians to establish the time of myocardial ischemic necrosis development. In the complicated forms, such as repeated and recurrent infarctions, the task to determine the time of infarction occurrence becomes more difficult.

There exist various methods of anatomicopathological diagnosis of myocardial ischemia. At the macroscopic level, tests with potassium tellurite and nitro blue tetrazolium are used to detect vanishing of enzymatic activity in ischemic areas.

At the microscopic level, special histological staining is used. Thus, Masson's trichrome staining is used to differentiate necrotic myocardium (blue staining) from the viable myocardial cytoplasm (red staining of the cytoplasm), often having a violet color of the area adjoining the zone of necrosis [2]. But it is impossible to establish how much time passed since the moment of necrosis development.
A histochemical method, and the reaction revealing the activity of phosphorylase, in particular, is considered to be one of the informative methods enabling the detection of early changes. However, application of this reaction in autopsy has not found a wide use because it is diagnostically significant only within $3 \mathrm{~h}$ after death [3].

As the molecular basis of cardiomyocyte activity became more explored, diverse immunohistochemical stains came into use: staining for the terminal complement complex C5b-9 [4], myoglobin, actin and tropomyosin [5], fibronectin [6], cardiac troponin I [7], heart-type fatty acid binding protein [8], staining for dystrophin [9].

To define the early stages of myocardial infarction, it is most advanced to use staining for desmin, which starts to disappear from the cytoplasm in the infarction zone $30 \mathrm{~min}$ after ischemia onset and is completely lost in 90-120 min [10].

Ortmann et al. (2000) examined the zone of myocardial ischemia for the depletion of such cardiac antigens as troponin $\mathrm{C}$ and $\mathrm{T}$, desmin, myoglobin, loss of

For contacts: Mikhail G. Shurygin, e-mail: mshurygin@gmail.com 
CD59, deposit of fibrinogen, fibronectin, and the terminal complement complex C5b-9.

C5b-9 complex was visualized in such tissues 30 min after the development of myocardial infarction symptoms. Depletion of cellular antigens started earlier than the precipitation of antigens from blood plasma [11].

Absence of "perfect" methods of morphological diagnosis of myocardial infarction has given us the idea to carry out new investigations in this field.

Given that the processes of myocardium remodeling are sharply activated immediately after the episode leading to necrosis, and this activity persists until the establishment of new interrelations of the cellular and interstitial components of myocardium, we have studied the feasibility of determining myocardial infarction age basing on the dynamics of the main participants of the connective-tissue framework reorganization, i.e. matrix metalloproteinases, including metalloproteinase 9 (MMP9).

The aim of the investigation was to develop a simple and available for practical application method of pathomorphological assessment of myocardial infarction age with a wide range of registered terms.

Materials and Methods. Experimental modeling of myocardial infarction was performed on 9-month old Wistar female rats weighing 220-250 g ( $=50)$ using diathermocoagulation of the periconical interventricular artery [12].

The experiment was carried out in compliance with the norms of humane animal treatment which are regulated by "The rules of conducting the works with experimental animals" (Supplement to the order No.755 of the Ministry of Health of the USSR of 12.08.1977) in accordance with ethical principles established by European Convention for the Protection of Vertebrate Animals used for Experimental and other Scientific Purposes (the Convention was passed in Strasbourg, March 18, 1986, adopted in Strasbourg, June 15, 2006), and approved by Biomedical Ethics Committee of the Irkutsk Scientific Center of Surgery and Traumatology.

The animals were withdrawn from the experiment in the period from $2 \mathrm{~h}$ to 30 days after myocardial infarction modeling. The heart of the experimental animal was fixed in $10 \%$ solution of neutral buffered formalin for $24 \mathrm{~h}$ at $20^{\circ} \mathrm{C}$ for further histochemical examination.

Post-mortem specimens of 30 men (29 with transmural myocardial infarction, 1 with postinfarction cardiosclerosis) were used as a material for pathomorphological investigation. There were 18 men and 12 women at the average age of 63.7 years. 16 patients died in the period within 3 days, 14 in the period from 3 days to 1 month. Autopsy was performed in the centralized prosectorium of the Irkutsk Regional Bureau of Morbid Anatomy receiving lethal cases from the Regional Clinical Hospital of Irkutsk city, which has a specialized cardioresuscitation unit. The investigation was approved by the Biomedical Ethics Committee of the Irkutsk Scientific Center of Surgery and Traumatology.
In 27 cases the diagnosis of acute myocardial infarction was established based on the clinical picture, electrocardiography data, reaction of cardiospecific enzymes, and confirmed at autopsy. In 3 cases the diagnosis of myocardial infarction was initially made at pathoanatomic autopsy. In 4 cases the diagnosis of acute myocardial infarction as one of the primary causes of death was combined with other nosological forms (stomach ulcer complicated by hemorrhage; tuberculosis; megaloblastic anemia; intestinal fistulas).

The assessment of myocardial infarction age was performed based on standard histological criteria: cardiomyocyte necrosis, neutrophilic infiltration and the character of its distribution, infiltration with lymphocytes, macrophages, plasmatic cells, proliferation of blood vessels, fibroblasts, collagen fibers. The tissue specimens from the myocardial infarction zone and the adjoining area were fixed with $10 \%$ neutral buffered formalin for $24 \mathrm{~h}$ at $20^{\circ} \mathrm{C}$ and embedded in paraffin blocks.

To stain for MMP9, a previously described immunohistochemical method was used [13]. Indirect peroxidase method was employed. Demasking and immunohistochemical staining were done manually using Novolink Polymer Detection System (Novocastra Laboratories Ltd., UK), 3,3'-diaminobenzidine (DAB) was used as a chromogen. Nuclei were stained with Mayer's hematoxylin.

Rabbit monoclonal antibodies (IgG) to MMP9 (Clone 1D:EP1254, Cat. No.2551-1, Lot YG 113001P; Epitomics Inc., USA) at dilution range from 1:100 to 1:250 were used as primary antibodies.

Results. At the first stage, the dynamics of staining the infarction zone and periinfarction zone for MMP9 was assessed in experimental myocardial infarction. MMP9 at high concentration is known to be present in neutrophils. During the investigation it was established that in the period from $2 \mathrm{~h}$ to 1 day after myocardial infarction modeling, brightly stained neutrophils in the vessel lumens in the infarction and periinfarction zones were detected (Figure $1(a)$ ).

On day 1, degranulation of neutrophils with the loss of their cytoplasm color was observed. Concurrently, there was detected appearance of the marked color of the extracellular matrix in the zone of ischemic damage which signified the release of MMP9 from neutrophils to the tissues (Figure 1 (b)).

On days $3-14$, a specific color of some fibroblastic cells appeared in the periinfarction zone in the experimental infarction. Availability of MMP9 in these cells at the indicated terms of the pathologic process is likely to be associated with the necessity of reorganization of the extracellular matrix (remodeling) in the new conditions of mechanical loads on the myocardium. Maximal color intensity of the cellular elements was noted on day 7-14 (Figure 1 (c)). On day 30 of the experimental infarction, specific color in the zone of postinfarction cardiosclerosis was not registered.

In the course of pathomorphological material 

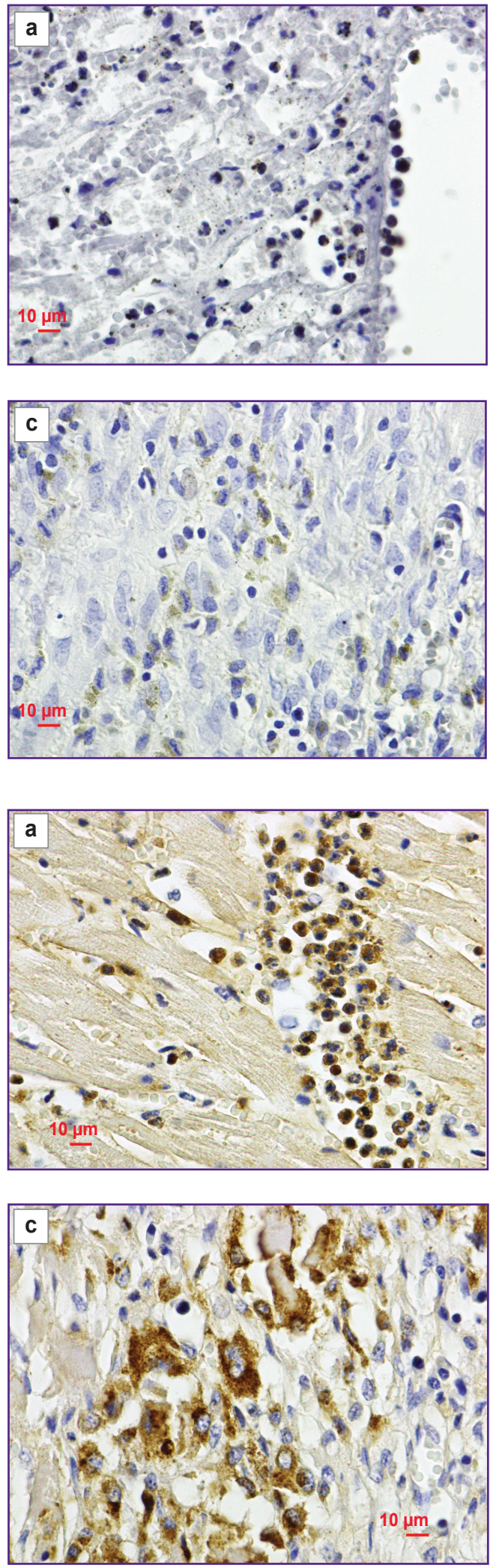

investigations, the dynamics of MMP9 content and localization in the myocardium of the dead patients was found to correspond to the data obtained during experimental studies. In lethal outcomes developed

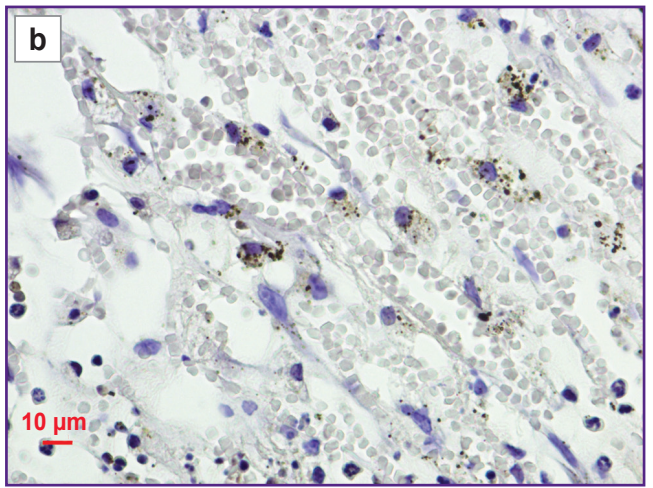

Figure 1. Immunohistochemical staining of the experimental material for MMP9 in the periinfarction zone:

(a) cytoplasmic expression in neutrophils after $12 \mathrm{~h}$; (b) staining of the extracellular matrix on day 1 ; (c) specific color of some cells of fibroblast line on day 7 . Indirect peroxidase method; DAB

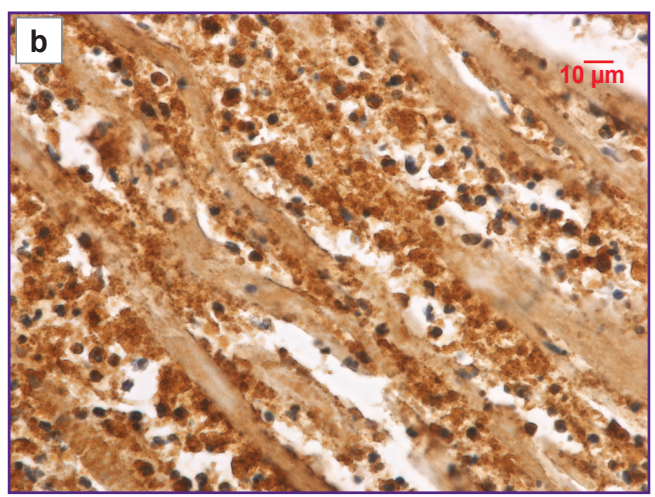

Figure 2. Immunohistochemical staining of the sectioned material for MMP9 in the periinfarction zone:

(a) brightly stained neutrophils after $9 \mathrm{~h}$; (b) a bright color of the extracellular matrix on day 2; (c) expression of MMP9 in the cells of fibroblast line on day 14 . Indirect peroxidase method; DAB

during several hours after infarction, brightly stained neutrophils in the vessels of periinfarction zone and in the zone of infarction were registered (Figure 2 (a)).

If infarction happened to occur $1-2$ days ago, neutrophil 
degranulation, loss of neutrophil cytoplasm staining were fixed. Along with this, a bright color of the extracellular matrix in the infarction zone was noted (Figure 2 (b)). In later terms (days 3-21) MMP9 expression in the cells of fibroblastic line was observed in the adjoining zone. In this case, the maximal color intensity was found on days 7-14 (Figure 2 (c)). The zone of formed cardiosclerosis (30 or more days) was not stained.

Thus, a good reproducibility of the proposed method has been noted: staining of multiple sections in different lots showed identical results.

Discussion. Staining of histological specimens of myocardium for MMP9 makes it possible to visualize the dynamics of infarction necrotic phase development and MMP9 release to the damaged zone in early phase of the pathologic process as well as functional activity of a number of cells in remodeling fibrillar proteins in the zone of postinfarction cardiosclerosis formation in later terms. At early stages, the main source of MMP9 in the damaged zone are neutrophils migrating to the focus of the ischemic injury during a neutrophilic phase of inflammation.

In later terms, staining of the cells of fibroblastic line for MMP9 with maximal intensity on days 7-14 reflects the remodeling processes in the zone of postinfarction cardiosclerosis formation as well as in the adjoining myocardial areas. It is known that myocardium remodeling occurring after the episode of ischemic damage has a very important aspect: reorganization of connective-tissue framework in accordance with the new conditions of mechanical load distribution [14]. The most important role in this process is given to the enzymes of metalloprotease group. The intensity of fibroclast staining in the zone of postinfarction cardiosclerosis formation reflects the activity of extracellular matrix reorganization. Reduction in the activity of the given process is accompanied by the decrease of intensity with the following loss of the specific color.

In connection with the clearly marked staging of MMP9 expression in the myocardium, it is convenient to use this marker for determining myocardial infarction age [15]. It also allows pathologists to perform pathomorphological differential diagnosis in repeated and recurrent myocardial infarctions.

Conclusion. The developed method to determine the time of myocardial infarction age enables pathologists to differentiate the time that passed since the infarction onset during pathomorphological investigations.

Study Funding. The work was supported by project financing of FSBSI ISCST (No.01201280990) and FSBIS ISC SB RAS.

Conflicts of Interest. The authors have no conflicts of interest to disclose.

\section{References}

1. Zech W.D., Schwendener N., Persson A, Warntjes M.J., Jackowski C. Postmortem MR quantification of the heart for characterization and differentiation of ischaemic myocardial lesions. Eur Radiol 2015; 25(7): 2067-2073, https:// doi.org/10.1007/s00330-014-3582-2.

2. Kostin S., Hein S., Arnon E., Scholz D., Schaper J. The cytoskeleton and related proteins in the human failing heart. Heart Fail Rev 2000; 5(3): 271-280, https://doi. org/10.1023/A:1009813621103.

3. Kakturskiy L.V., Rybakova M.G., Kuznetsova I.A. Sudden cardiac death (morphological diagnosis). Biblioteka patologoanatoma 2008; 100: 45-61.

4. Thomsen H., Held H. Immunohistochemical detection of $\mathrm{C} 5 \mathrm{~b}-9(\mathrm{~m})$ in myocardium: an aid in distinguishing infarctioninduced ischemic heart muscle necrosis from other forms of lethal myocardial injury. Forensic Sci Int 1995; 71(2): 87-95, https://doi.org/10.1016/0379-0738(94)01640-q.

5. Campobasso C.P., Dell'Erba A.S., Addante A., Zotti F., Marzullo A., Colonna M.F. Sudden cardiac death and myocardial ischemia indicators: a comparative study of four immunohistochemical markers. Am J Forensic Med Pathol 2008; 29(2): 154-161, https://doi.org/10.1097/PAF.0b013e318177eab7.

6. Hu B.J., Chen Y.C., Zhu J.Z. Study on the specificity of fibronectin for post-mortem diagnosis of early myocardial infarction. Med Sci Law 2002; 42(3): 195-199, https://doi. org/10.1177/002580240204200303.

7. Sapouna R., Gourgiotis D., Athanaselis S., Papadodima S., Spiliopoulou C. Diagnostic value of cardiac troponin I in postmortem diagnosis of myocardial infarction. Am J Forensic Med Pathol 2013; 34(2): 139-141, https://doi. org/10.1097/PAF.0b013e3182880aa1.

8. Meng X., Ming M., Wang E. Heart fatty acid binding protein as a marker for postmortem detection of early myocardial damage. Forensic Sci Int 2006; 160(1): 11-16, https://doi.org/10.1016/j.forsciint.2005.08.008.

9. Hashmi S., Al-Salam S. Loss of dystrophin staining in cardiomyocytes: a novel method for detection early myocardial infarction. Int J Clin Exp Pathol 2013; 6(2): 249-257.

10. Ouyang J., Guzman M., Desoto-Lapaix F., Pincus M.R., Wieczorek R. Utility of desmin and a Masson's trichrome method to detect early acute myocardial infarction in autopsy tissues. Int J Clin Exp Pathol 2009; 3(1): 98-105.

11. Ortmann C., Pfeiffer H., Brinkmann B. A comparative study on the immunohistochemical detection of early myocardial damage. Int J Legal Med 2000; 113(4): 215-220, https://doi.org/10.1007/s004149900094.

12. Shurygin M.G., Shurygina I.A., Dremina N.N. Dynamics of vasoendothelial growth factor and fibroblast growth factor in experimental cardiac infarction. Byulleten' VostochnoSibirskogo nauchnogo tsentra Sibirskogo otdeleniya Rossiyskoy akademii meditsinskikh nauk 2007, 6. 169-114.

13. Shurygina I.A., Shurygin M.G., Ayushinova N.I. Expression of apoptosis markers in adhesions in the abdominal cavity under the experimental conditions. Vestnik Rossiyskoy akademii meditsinskikh nauk 2014, 69(5-6): 29-33.

14. Ruiz-Villalba A., Simon A.M., Pogontke C. Castillo M.I., Abizanda G., Pelacho B., Sánchez-DominguezR. Segovia J.C., Prósper F., Pérez-Pomares J.M. Interacting resident epicardiumderived fibroblasts and recruited bone marrow cells form myocardial infaretion scar. J Am Coll Cardio 2015; 65(19): 2057-2066, https:Hdoi.org/10.1016/j.jacc.2015.03.520.

15. Shurygina I.A., Shurygin M.G., Dremina N.N., Kanya O.V. Sposob patomorfologicheskogo opredeleniya davnosti nastupleniya infarkta miokarda [Method for pathomorphological determination of prescription of myocardial infarction]. Patent RU 2518333 C1. 2012. 